\title{
Use of virtual assisted lung mapping (VAL-MAP), a bronchoscopic multispot dye-marking technique using virtual images, for precise navigation of thoracoscopic sublobar lung resection
}

\author{
Masaaki Sato, MD, PhD, Mitsugu Omasa, MD, PhD, Fengshi Chen, MD, PhD, Toshihiko Sato, MD, PhD, \\ Makoto Sonobe, MD, PhD, Toru Bando, MD, PhD, and Hiroshi Date, MD, PhD
}

\begin{abstract}
Objective: We have developed a novel bronchoscopic multiple marking technique to assist resection of hardly palpable lung tumors. Because 3-dimensional virtual images were used and multiple markings made on the lung surface to provide "geometric" information, we termed this technique "virtual assisted lung mapping" (VAL-MAP). The safety and efficacy of VAL-MAP were evaluated.
\end{abstract}

\begin{abstract}
Methods: Virtual bronchoscopy was used to select 2 to 4 appropriate bronchial branches for marking. Bronchoscopy was conducted with the patient under local anesthesia. A metal-tip catheter was inserted into a selected bronchus and advanced to the pleura. The location of the catheter tip was fluoroscopically confirmed, and 1 $\mathrm{mL}$ of indigo carmine was injected. This procedure was repeated to complete all the planned markings. Post-VAL-MAP computed tomography was used to visualize the localization of the multiple markings on 3 -dimensional virtual images, which were used as references in the subsequent operation.
\end{abstract}

Results: Of the 95 marking attempts made for 37 tumors in 30 patients, 88 (92.6\%) were identified and contributed to the surgery. No clinically evident complications were associated with the procedure. A total of 15 wedge resections and 18 segmentectomies were thoracoscopically conducted, with a successful resection rate of $100 \%$. Multiple markings of the VAL-MAP were complementary, enabling us to achieve complete resection even when 1 of the markings failed. The markings were visible even on interlobar fissures, at the apex, and on the diaphragm, which conventional percutaneous marking can hardly reach.

Conclusions: VAL-MAP was safely conducted with satisfactory outcomes in our early experience. Additional confirmation of its safety and efficacy is necessary. (J Thorac Cardiovasc Surg 2014;147:1813-9)

Sublobar lung resection (wedge resection and segmentectomy) for small tumors is often challenging because of the small size and minimal palpability of the tumor. Less invasive video-assisted thoracic surgery (VATS) has made tumor identification even more difficult. Even if a tumor is identifiable, it is crucial to obtain a secure surgical margin, especially around the hardly palpable ground glass opacity (GGO) components. An efficient intraoperative navigation system could play an important role in overcoming these challenges.

Various strategies have been reported to identify small pulmonary lesions. ${ }^{1-10}$ However, percutaneous computed tomography (CT)-guided marking has been technically limited by the presence of anatomically inaccessible locations such as the lung apex. ${ }^{1-7,10}$ Moreover, although the hook wire method has reportedly been generally

\footnotetext{
From the Department of Thoracic Surgery, Kyoto University Hospital, Kyoto, Japan. Disclosures: Authors have nothing to disclose with regard to commercial support. Received for publication March 30, 2013; revisions received Nov 8, 2013; accepted for publication Nov 22, 2013; available ahead of print Jan 31, 2014.

Address for reprints: Masaaki Sato, MD, PhD, Department of Thoracic Surgery, Kyoto University Hospital, 54 Kawahara-chyo, Shyogoin, Sakyo-ku, Kyoto 606-8507, Japan (E-mail: satomasa@kuhp.kyoto-u.ac.jp). $0022-5223 / \$ 36.00$

Copyright (c) 2014 by The American Association for Thoracic Surgery http://dx.doi.org/10.1016/j.jtcvs.2013.11.046
}

successful, multiple risks have been reported, including pneumothorax, bleeding, dislodgement and loss of the marker, ${ }^{1-3,10}$ and, most concerning, life-threatening air embolism with subsequent cerebral or cardiac infarction. ${ }^{11-16}$ Although CT-guided bronchoscopic transairway lung marking ${ }^{17-20}$ has been less concerning with respect to these safety issues, such procedures have not been commonly conducted because of the difficulties associated with the settings and the extended radiation exposure.

To overcome the limitations of conventional lung marking techniques and to realize efficient intraoperative navigation for thoracoscopic lung resection, we report a new strategy termed "virtual assisted lung mapping" (VAL-MAP). This simple technique uses 3-dimensional (3D) images and bronchoscopic dye injection under conventional fluoroscopy to achieve simultaneous multiple marking (lung mapping). In addition to the purpose of conventional lung marking to localize tumors, the "geometric" information on the lung surface provided by VAL-MAP can navigate thoracoscopic sublobar lung resection and secure sufficient resection margins.

\section{METHODS}

\section{Patient Selection}

The patients were selected for VAL-MAP according to 1 of the following criteria: a pulmonary lesion that was expected to be hardly 


$$
\begin{aligned}
& \text { Abbreviations and Acronyms } \\
& \begin{aligned}
\text { 3D } & =3 \text {-dimensional } \\
\text { CT } & =\text { computed tomography } \\
\text { GGO } & =\text { ground glass opacity } \\
\text { VATS } & =\text { video-assisted thoracic surgery }
\end{aligned}
\end{aligned}
$$

palpable; a pulmonary lesion that should be resected by wedge resection or segmentectomy, necessitating careful determination of the resection margins because of the tumor location and/or extension of the GGO component; or severe pleural adhesions expected to interfere with tumor identification. Patients with significant pleural adhesions were included because even a solid tumor of reasonable size could become hardly identifiable in such cases and intraoperative disorientation could easily occur, especially during VATS. Patients with a medical history of bronchial asthma were excluded. All patients provided consent for inclusion in the present study. The ethics committee of the Kyoto University Graduate School and Faculty of Medicine examined and approved the study protocol.

\section{VAL-MAP Design}

VAL-MAP was conducted according to the steps shown in Figure 1. VAL-MAP was designed with 2 to 3 markings for wedge resections and 3 to 4 markings for segmentectomy. Representative cases of wedge resection and segmentectomy are shown in Figures 2 and 3, respectively. The location of the markings was designed to provide "geometric" information on the lung surface to help with tumor identification and determination of the resection lines. 3D virtual bronchoscopy was constructed from thin-slice CT images using computer software (Aquarius iNtuition Client Viewer; TeraRecon, Inc, Tokyo, Japan), and the target bronchial branches were manually identified. The marking plan was also reviewed by constructing 3D images. For suspected lung cancer with a solid component on CT scans or pure GGO lesions $>1 \mathrm{~cm}$ in diameter, segmentectomy was selected, and VAL-MAP was designed to obtain a resection margin of $>2 \mathrm{~cm}$. For pure GGO lesions $<1 \mathrm{~cm}$ in diameter or suspected metastatic tumors, segmentectomy or wedge resection was selected, depending on the tumor size and location. VAL-MAP was designed to obtain a resection margin of $>1.5 \mathrm{~cm}$ in these cases.

\section{Marking Procedure}

Marking procedures were conducted within 24 hours before each operation in a bronchoscopy suite equipped with fluoroscopy. The timing of the procedure was determined according to logistical reasons. The steps of the marking procedures have been described on the right side of Figure 1. Laryngopharyngeal local anesthesia was established using $4 \%$ lidocaine spray, and this was followed by intravenous sedation with 2 to $3 \mathrm{mg}$ of midazolam. Sedation was maintained at the level at which the patient could respond when their name was called. A regular flexible bronchoscope (BF 260; Olympus, Tokyo, Japan) was orally inserted. A metal-tip bronchoscopic spray catheter (PW-6C-1, Olympus) was preloaded with $1 \mathrm{~mL}$ of indigo carmine (Daiichi-Sankyo Inc, Tokyo, Japan). After identification of a target bronchus using virtual bronchoscopic guidance, the catheter was inserted through the working channel of the bronchoscope into the target bronchus. The direction of the catheter was confirmed fluoroscopically, and the catheter was advanced further to reach the visceral pleura (Figure 2, C). The 3-way stopcock connecting the catheter and a syringe containing air ( 10 or $30 \mathrm{~mL}$ ) was then opened. At this point, the catheter tip was usually wedged, and resistance was felt through the plunger if it was gently pushed. The plunger was never forcefully pushed further. The catheter was slowly and continuously withdrawn while feeling the resistance on the plunger until the point at which the resistance suddenly decreased, and the plunger could be easily pushed. Fluoroscopy was turned off, and 30 to $90 \mathrm{~mL}$ of air per mark was gently injected. The catheter was then withdrawn. This process was repeated for all targeted bronchi.

\section{Post-VAL-MAP CT and 3D Image Construction}

After bronchoscopic marking, thin-slice CT was performed within 3 hours to examine the localization of the actual markings. Although indigo carmine is not radiopaque, the markings were identifiable on the CT scan as a GGO-like attenuation, with or without some bronchial dilatation, presumably because of creation of artifact similar to that observed after bronchoalveolar lavage (ie, water in the lung parenchyma; Figure 2, D). 3D lung images that included the markings and a target lesion were constructed (Figures 2, E, and 3,C). Using this information, the final lung resection strategy was adjusted (interrupted lines in Figures 2, E, and 3, $C$ ). In cases of planned segmentectomy, the $3 \mathrm{D}$ images were also used to predetermine the hilar structures to be resected (pulmonary arteries and veins and bronchi).

\section{Surgical Resection}

VATS was used for all surgical resections. For wedge resection, a stapler was primarily used along the resection line planned by VAL-MAP. Electrocautery was also used if necessary, depending on the tumor location and surgeon preference. For segmentectomy, the hilar structures were dissected and resected according to the preoperative plan. Resection lines were made using electrocautery or a stapler, or both. The segmentectomy procedures were either conventional (guided by intersegmental veins) or unconventional (subsegmentectomy, pulmonary artery-guided segmentectomy, ${ }^{21}$ or extended segmentectomy that went beyond the anatomic segment into an adjacent segment ${ }^{22}$ ).

\section{Statistical Analysis}

Data are expressed as the mean \pm standard deviation, as appropriate. No statistical analyses were performed throughout the study.

\section{RESULTS \\ VAL-MAP Design and Preparation}

From August 2012 to March 2013, 37 lesions in 30 patients were scheduled to undergo resection using VAL-MAP. The characteristics of the patients and lesions and a list of the selected procedures are presented in Table 1. VAL-MAP was conducted for expected pleural adhesion in 4 cases: reoperation in 2, previous pleurodesis in 1 , and a history of tuberculous pleuritis in 1. A total of 95 lung markings were planned. The VAL-MAP design, using virtual bronchoscopy, required 20 minutes to 1 hour, depending on the number of markings and the familiarity of the surgeon and/or bronchoscopist with the 3D software.

\section{Marking Procedure}

The marking procedure was conducted on the same day of surgery for 70 markings of 23 patients, and 25 markings of 7 patients were performed the day before surgery for logistical reasons. A total of 95 marking attempts were made among the 30 patients using VAL-MAP. One marking took an average of 2 to 3 minutes, and the whole procedure required 5 to 15 minutes per patient, depending on the number of markings and the difficulty in inserting the catheter. 


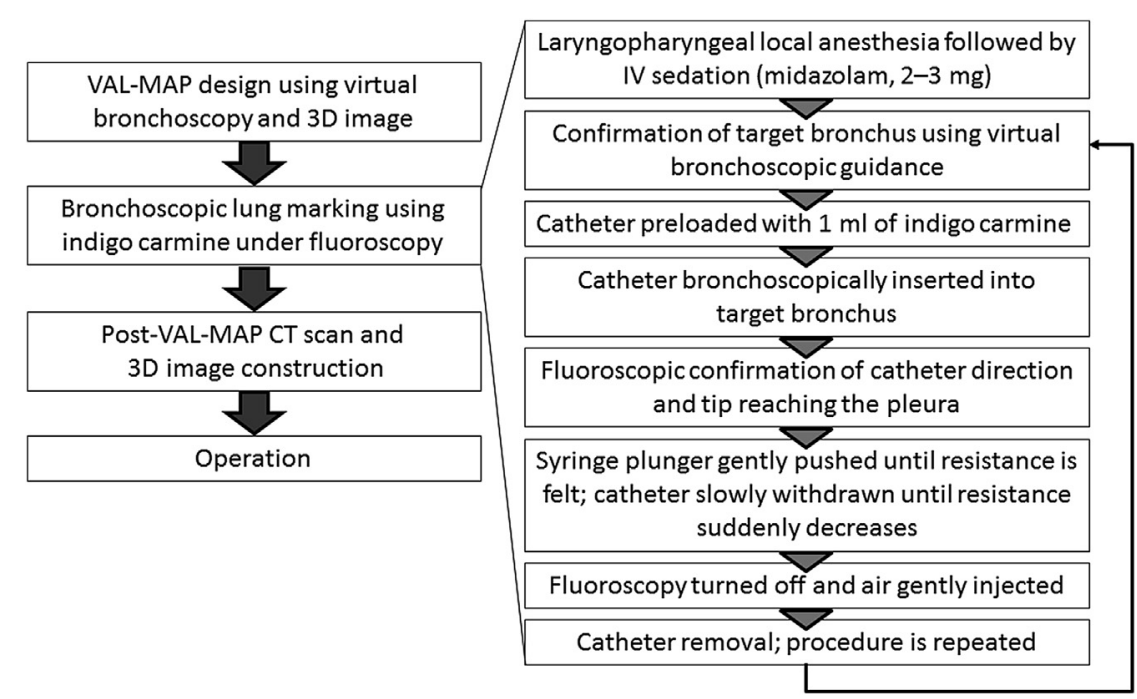

FIGURE 1. Steps of virtual assisted lung mapping (VAL-MAP). CT, Computed tomography; $3 D$, 3-dimensional.

The fluoroscopic radiation exposure time was 20 to $45 \mathrm{sec}-$ onds per mark, and the total radiation time was limited to 1 to 2 minutes per patient. No clinically evident complications were associated with the marking procedure. The clinically silent complications included intra-alveolar bleeding in 1 (minor temporal bleeding during bronchoscopy that was visible as limited infiltration on the CT scan), a tiny bulla found intraoperatively beneath the visceral pleura in 1 , and minimal pneumothorax found on postmarking CT scan in 2 .

\section{Outcome of VAL-MAP}

Of the 95 marking attempts, 91 were visible during the operation $(95.7 \%)$. The markings attempted the day before surgery were as clearly visible during the operation as those performed the same day. In patients with significant pleural adhesions, the markings were clearly visible and were helpful for establishing orientation, identifying the target lesion, and obtaining a secure surgical margin. Of the 4 failed markings, 1 was found in the relatively central airway on the postmarking CT scan, suggesting that the catheter had not reached the visceral pleura. In another case, the location of the marking appeared to be overlapping with dense pleural anthracosis. No clear explanation was found for the other 2 failed markings. Of the 91 visible markings, 3 were considered unsuccessful. In 2 of these 3 patients, a marking was mistakenly conducted through a small bronchial branch that was not visualized on $3 \mathrm{D}$ virtual bronchoscopy, leading to localization of the marking too far from the original plan to assist in surgery; in the third patient, the postmarking CT findings were misinterpreted because of artifact that had probably been created during the marking procedure. The remaining 88 markings $(92.6 \%)$ were visible and believed to be helpful for surgical resection.

\section{Outcome of Surgical Resection}

All surgical procedures were completed using VATS, and all 37 lesions were successfully resected with satisfactory resection margins. During segmentectomy for suspected lung cancer with a solid component or a pure GGO lesion of $>1 \mathrm{~cm}$ in diameter, a resection margin of $>1.5 \mathrm{~cm}$ in collapsed lungs was confirmed intraoperatively. During segmentectomy or wedge resection of pure GGO lesions $>1 \mathrm{~cm}$ in diameter or suspected metastatic tumors, a resection margin $>1.0 \mathrm{~cm}$ was confirmed if the tumors were identifiable intraoperatively. A total of 21 lesions were not palpable during surgery, even after extraction of the specimen. However, 12 of these lesions were identified during surgery by cutting the specimen according to the mapping information, and the existence of the lesion was confirmed by frozen section analysis. A sufficient resection margin was then confirmed visually. In the remaining impalpable lesions, the localization and resection margin were estimated according to the mapping information; these lesions were eventually identified pathologically, again, according to the mapping information. The final pathologic entity of these lesions is summarized in Table 1.

Although one of the multiple markings failed in $7 \mathrm{pa}-$ tients, as described, the other markings functioned as a sufficient "map" to guide the surgery in these cases. The surgeons did not have to switch from wedge resection to segmentectomy or from segmentectomy to lobectomy because of unreliable markings that could have necessitated extended resection for complete removal of the target lesions. The total operation time and interval from the initiation of the operation to extraction of the specimen was 55 \pm 14 and $21 \pm 6$ minutes for single wedge resection $(\mathrm{n}=7)$ and $190 \pm 43$ and $119 \pm 35$ minutes for single segmentectomy $(\mathrm{n}=20)$. The operations that included $>2$ resections were excluded. Surgical complications included 

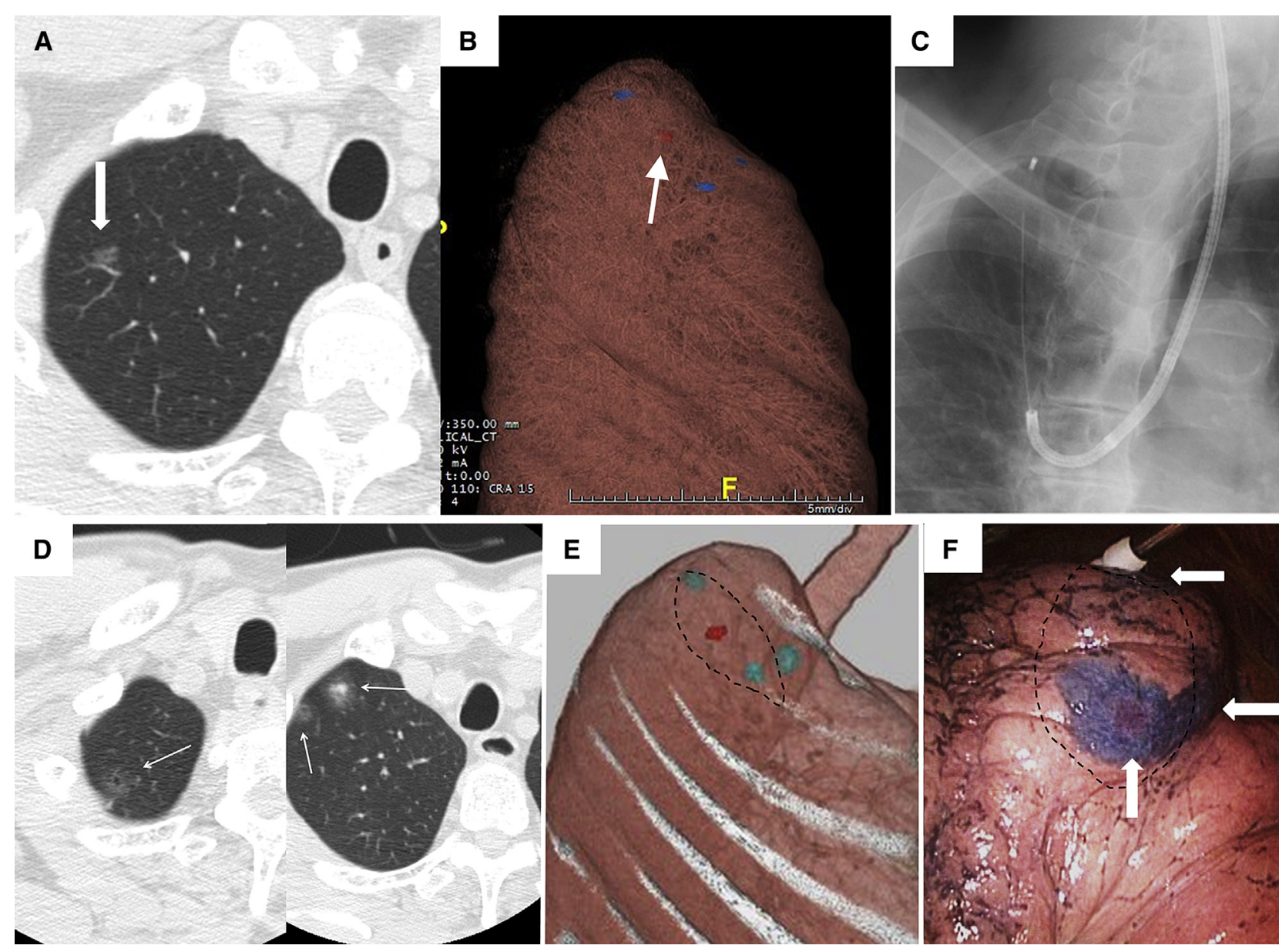

FIGURE 2. Conduction of virtual assisted lung mapping (VAL-MAP). A, Computed tomography (CT) scan of a patient who was found to have a ground glass opacity lesion ( $8 \mathrm{~mm}$ in diameter) in the right upper lobe during chest CT screening. B, Three-dimensional view of the VAL-MAP plan. The target lesion (red) and 3 planned markings (blue) are shown. C, Fluoroscopic view of the metal-tip catheter inserted into a target bronchus and reaching the pleura. D, CT scan taken 1 hour after VAL-MAP showing 3 markings as new ground glass opacity-like changes (arrows). E, Three-dimensional image constructed from post-VAL-MAP CT scan shown in Figure D. The target lesion (red) and 3 actual markings (blue) are shown. The interrupted line indicates the planned resection line by a stapler. In this case, the apical and lower lateral markings would have been included in the resection specimen, with the lower medial marking used as a reference to obtain the lateral resection margin, which would at the same time provide information regarding the depth of resection when a stapler was used. F, Intraoperative thoracoscopic view showing 3 blue markings on the lung surface (arrows). The interrupted line indicates the actual resection line. Permanent pathologic examination demonstrated carcinoma in situ.

intraoperative bleeding during repeat thoracotomy in 1, a persistent intrathoracic space after segmentectomy in 1, and atelectasis in the lingular segment after left superior segmentectomy in 1 . None of them were attributed to VAL-MAP.

\section{DISCUSSION}

We have reported our early experience with a novel bronchoscopic multiple marking technique, VAL-MAP. This technique is characterized by the following features. First, the marking procedure is planned using $3 \mathrm{D}$ lung images and virtual bronchoscopy to precisely guide the later bronchoscopic marking procedures without real-time CT guidance. Second, bronchoscopic lung marking is performed by injecting indigo carmine under regular fluoroscopy without puncturing the lung to minimize the risk of air embolism. Finally, multiple markings can help surgeons, not only to identify the target lesion, but also to obtain a sufficient resection margin by providing "geometric" information on the lung surface. In the 95 attempted markings for 37 lesions in 30 patients using VAL-MAP, no clinically evident adverse events were experienced, and all targeted lesions were resected successfully.

Percutaneous CT-guided lung marking has technical limitations and safety concerns. For the hook wire method, multiple risks have been reported, including pneumothorax $(32.1 \%)$, bleeding $(14.9 \%)$, hemothorax $(0.6 \%)$, and dislodgement and loss of the marker ( $\leq 5 \%$ of cases). ${ }^{2}$ Similarly, critical air embolism has also been reported with percutaneous CT-guided lung biopsy at a rate of 


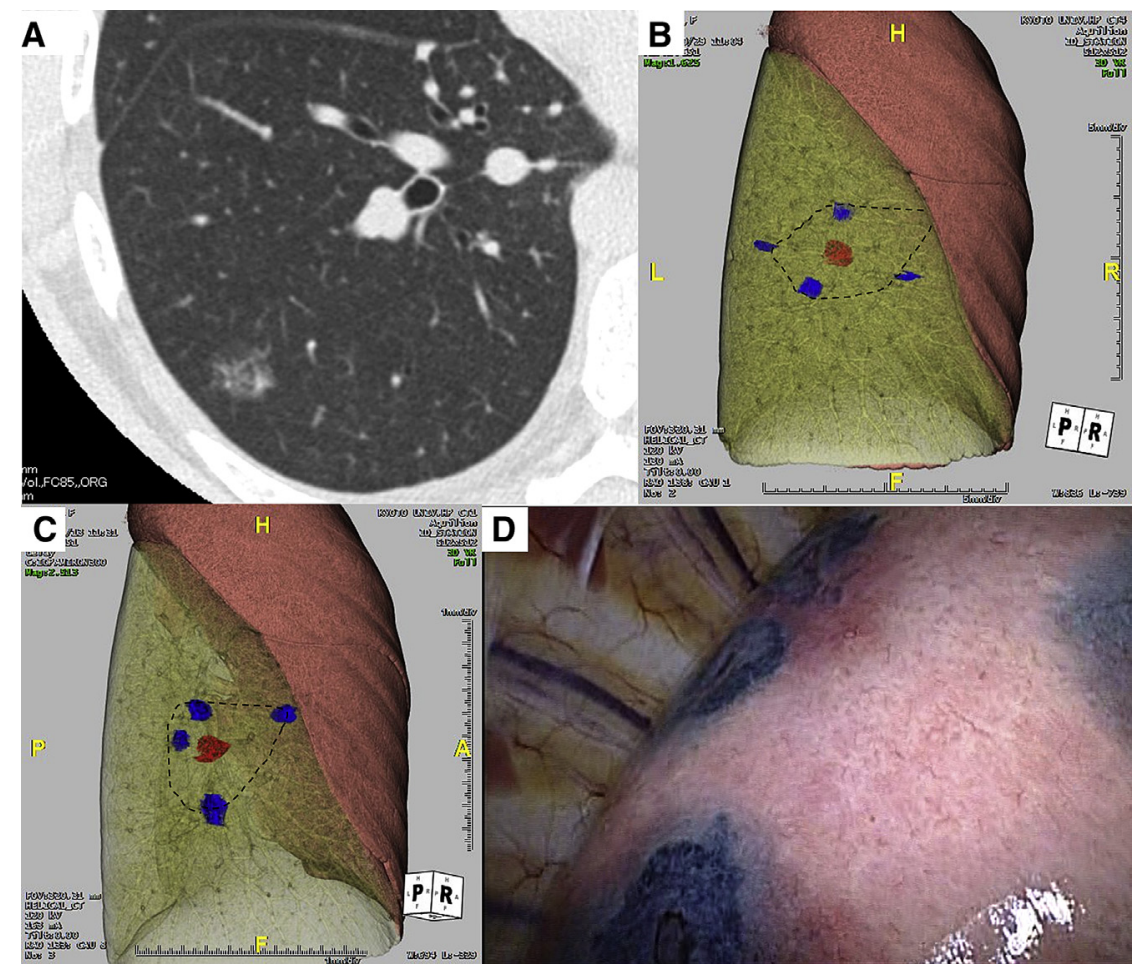

FIGURE 3. Representative case of virtual assisted lung mapping (VAL-MAP) used for segmentectomy. A, A ground glass opacity lesion of 9 mm in diameter found in the right lower lobe. Resection of the superior subsegment of the posterolateral basal segment was planned. B, VAL-MAP plan with 4 markings indicating the location of the target lesion and intersegmental resection lines. C, Post-VAL-MAP 3-dimensional image constructed from a thin-slice computed tomography (CT) scan taken after VAL-MAP. Note, the localization of the actual markings was slightly away from the original plan in Figure B but still provided information regarding the location of the target lesion and resection lines. Thus, the operation plan was adjusted according to the "actual" markings after VAL-MAP. D, Intraoperative thoracoscopic view showing 4 blue markings on the lung surface. Note, the relationships among the markings were identical to those observed on the post-VAL-MAP 3-dimensional image in Figure C. The blue dots in Figures B and C indicate markings; the red dot indicates the target ground glass opacity lesion. The interrupted lines indicate the planned resection line. Permanent pathologic examination demonstrated stage T1a adenocarcinoma with a free resection margin.

$0.05 \%$ to $0.07 \%{ }^{23}$ In contrast, VAL-MAP had a low risk of pneumothorax ( $6.6 \%$ per case; $2.1 \%$ per mark) with no hemothorax. Also, no concern exists regarding dislodgement of the instrument. Although more cases are needed, no air embolism developed in our series or other series of bronchoscopic marking. ${ }^{17-20}$ Moreover, theoretically, bronchoscopic techniques have no anatomic limitations regarding accessibility-the lung apex, area behind the scapula, and, even, interlobar fissures are accessible.

Furthermore, VAL-MAP possesses the advantages of bronchoscopic marking techniques. ${ }^{17-20}$ We also successfully introduced 3D lung images and virtual bronchoscopy to precisely guide later bronchoscopic marking procedures without real-time CT guidance, avoiding special settings in a CT room and excessive radiation exposure. Because of this simplicity, multiple markings (ie, mapping) were easily achieved within a limited period.

The primary purpose of VAL-MAP is to identify lesions expected to be hardly identifiable. A variable sensitivity of $50 \%$ to $100 \%$ has been reported for each method (ie, hook wire, dye, and radio-guided). ${ }^{10}$ One of the largest reports on a CT-guided wire method showed a resection success rate of 117 of 125 lesions among 108 patients $(93.6 \%))^{5}$ Although a simple comparison would not be appropriate because of different patient cohorts and surgical approaches, it is notable that our strategy using VAL-MAP resulted in a $100 \%$ resection success rate, including a number of subcentimeter lesions.

Importantly, the high resection success rate in our cohort could not simply be attributed to the success of a single marking procedure but to the multiple marking strategy we undertook. Of the 95 marking attempts, 91 markings were visible during surgery $(95.7 \%)$, and 88 markings $(92.6 \%)$ were thought to be truly helpful for surgical resection. However, the multiple markings in VAL-MAP allowed some room for technical failure. First, even if one of the markings failed, the other markings were still helpful to guide surgery; thus, multiple marking worked as "insurance" for satisfactory resection. Although we experienced several failures in marking, the remaining markings enabled successful complete resection. Second, visualization of the actual markings on the post-VAL-MAP CT scans and their 
TABLE 1. Patient and lesion characteristics

\begin{tabular}{|c|c|c|c|c|c|c|c|c|}
\hline $\begin{array}{l}\text { Radiographic } \\
\text { characteristics }\end{array}$ & $\begin{array}{l}\text { Patients } \\
\text { (n) }\end{array}$ & $\begin{array}{c}\text { Gender } \\
(\mathrm{M} / \mathrm{F})\end{array}$ & Age $(y)$ & $\begin{array}{l}\text { Lesions } \\
\text { (n) }\end{array}$ & $\begin{array}{l}\text { Selected operation } \\
\quad \text { (patients [n]) }\end{array}$ & $\begin{array}{l}\text { Lesion size } \\
\quad(\mathbf{m m})\end{array}$ & $\begin{array}{c}\text { Marks } \\
\text { (n)/patient }\end{array}$ & Pathologic findings (lesions) \\
\hline \multirow[t]{3}{*}{ Solitary pure GGO } & 17 & $5 / 12$ & $62.6 \pm 11.4$ & 17 & Wedge (4) & $6.7 \pm 1.3$ & $2.8 \pm 0.5$ & Tis (3), fibrosis (1) \\
\hline & & & & & Conv seg (8) & $11.0 \pm 2.2$ & $3.9 \pm 0.4$ & Tis (2), T1a AD (5), T1b AD (1) \\
\hline & & & & & Unconv seg (5) & $10.8 \pm 5.4$ & $3.8 \pm 0.4$ & Tis (1), T1a AD (4) \\
\hline \multirow[t]{2}{*}{ Two pure GGO } & 2 & $0 / 2$ & 50,52 & 4 & Wedge $\times 1 *(1)$ & 4,7 & 3 & Tis (1) + T1a AD (1) Fibrosis (1) \\
\hline & & & & & Wedge $\times 2(1)$ & 4,7 & 3 & + Tis $(1)$ \\
\hline Three pure GGO & 1 & $0 / 1$ & 63 & 3 & Unconv seg* (1) & $4,6,15$ & 4 & Tis $(2)+$ T1a AD (1) \\
\hline \multirow[t]{3}{*}{ Solitary mixed GGO ${ }^{\dagger}$} & 4 & $3 / 1$ & $64.0 \pm 3.7$ & 4 & Wedge (1) & 12 & 2 & $\mathrm{~T} 1 \mathrm{a} \mathrm{AD}(1)$ \\
\hline & & & & & Conv seg (2) & 16,24 & 2,2 & T1a AD (1), T1b Ad (1) \\
\hline & & & & & Unconv seg (1) & 16 & 2 & $\mathrm{~T} 1 \mathrm{a} \mathrm{AD}(1)$ \\
\hline Mixed and pure GGO & 1 & $0 / 1$ & 71 & 2 & Unconv seg* (1) & 13,6 & 3 & Tis (1) + T1a AD (1) \\
\hline \multirow[t]{2}{*}{ Solitary nodule } & 3 & $3 / 0$ & $64.0 \pm 1.0$ & 3 & Wedge (2) & 8,11 & 2,2 & T1a AD (1), Met colon Ca (1) \\
\hline & & & & & Conv seg (1) & 12 & 3 & $\mathrm{~T} 1 \mathrm{a} \mathrm{AD}(1)$ \\
\hline Two nodules & 2 & $2 / 0$ & $46.5 \pm 20.5$ & 4 & Wedge $\times 2(2)$ & $5.0 \pm 2.2$ & 4,4 & Met sarcoma (4) \\
\hline Total & 30 & $13 / 17$ & $63.4 \pm 10.9$ & 37 & $\begin{array}{l}\text { Wedge (14) } \\
\text { Conv seg (11) } \\
\text { Unconv seg (8) }\end{array}$ & $9.3 \pm 4.7$ & $3.2 \pm 0.9$ & $\begin{array}{c}\text { Fibrosis (2), Tis (11), T1a AD } \\
\text { (17), T1b AD (2), Met (5) }\end{array}$ \\
\hline
\end{tabular}

Data presented as mean \pm standard deviation or number of patients or lesions, as appropriate. $M$, Male; $F$, female; $G G O$, ground glass opacity (lesion); Wedge, wedge resection; Conv seg, conventional segmentectomy; Unconv seg, unconventional segmentectomy; $A D$, adenocarcinoma; $M e t$, metastatic; Ca, carcinoma. * Multiple lesions were resected in a single specimen. †Lesions containing both GGO and solid components.

3D image reconstruction allowed for misplacement of the markings. Because the catheter direction can be controlled only to the fifth to sixth bronchial branches, the markings can be diverted from the original plan; however, this would still be acceptable in most cases, because the surgical plan can be adjusted according to the actual markings depicted on the post-VAL-MAP CT scan.

Using conventional marking techniques, infrequent positive resection margins and recurrence at the surgical stump have been reported. ${ }^{3,5}$ Even if a marking has been successful in the identification of a tumor, the marker does not inform the surgeon of how extended the resection should be. In contrast, the multiple marking strategy of VAL-MAP can help surgeons to obtain a secure resection margin. During wedge resection, VAL-MAP could indicate how far and deep a stapler should be placed. For example, in Figure 2, $E$, the imaginary staple line was designed by considering the size of the GGO lesion. The 2 markings at both ends of the staple line indicated where to start and finish stapling, and the other marking indicated how much lateral margin should be taken and, thus, the depth of the lung tissue to be resected. Similarly, during segmentectomy, VAL-MAP helped to obtain a safe resection margin, especially when the tumor was located close to the intersegmental line and the resection needed to be extended into an adjacent segment (extended segmentectomy ${ }^{22}$ ).

The present patient cohort was our very first experience. However, in a preclinical study, we refined the bronchoscopic marking technique using dogs (data not shown). We found that the injection of air after the indigo carmine enabled vivid visualization of the markings by efficiently spraying the dye against the peripheral lung tissue. To avoid excessive pressure on the peripheral lung tissue, we found it important to withdraw the catheter to the point at which the catheter tip was unwedged, and extra air could then escape through the side of the catheter. In the first 20 cases, we experienced 2 cases of minor pneumothorax; we subsequently reduced the amount of air from 90 to $30 \mathrm{~mL} / \mathrm{mark}$. Gentle catheter insertion and manipulation was also important to avoid complications such as pneumothorax and intra-alveolar bleeding.

Through early experiences using VAL-MAP, we identified the tips and pitfalls of the technique. First, confirmation of the location of the metal tip under fluoroscopy at dye injection is key to successful marking. In an early case, we identified the marking in the relatively central airway on post-VAL-MAP CT scan; however, the marking was invisible during surgery. Since that experience, we have routinely rotated the fluoroscope to confirm the catheter tip is located at the periphery of the lung (Figure 2, C). Second, once the catheter has been inserted distally into the airway, dye injection should be completed without repeating catheter withdrawal and insertion. We found that repeated manipulation of the catheter could create an artifact in the lung, which could lead to misinterpretation of the post-VAL-MAP CT results.

We acknowledge that the present VAL-MAP technique has multiple limitations and, thus, room for technical improvement. First, improvement in the injection catheter could allow for more accurate marking using a thinner bronchoscope. The use of navigational bronchoscopy would help to guide a bronchoscope to more peripheral bronchial branches for more accurate marking. Second, indigo carmine could be mixed with or replaced by contrast 
material to better visualize the markings during CT. Third, the multiple steps necessary to complete the procedure could be shortened by introducing a hybrid operation room in which cone-beam CT is usable with the patient under general anesthesia. Nevertheless, although these technical issues could be overcome in the near future, we would emphasize that we intended to make the present technique feasible using only materials and equipment already commercially available and less costly. The catheter cost is in the range of US\$300 and reusable. Although the cost for imaging software will vary, free software is available for use during VAL-MAP. We believe that the present technique will be widely applicable in many institutions.

\section{CONCLUSIONS}

We developed a novel bronchoscopic multiple marking technique using 3D virtual images and termed it "VAL-MAP." The "lung map" can help identify the lesion and design secure resection lines in thoracoscopic operations. The technique is theoretically safer than conventional percutaneous techniques and has been free of clinically evident complications in our early experience. Additional accumulation of cases is required to establish the safety and usefulness of the technique.

The authors gratefully thank Drs Akihiro Aoyama, Tetsu Yamada, Toshi Menju, Masaki Ikeda, Yuhei Yokoyama, and Fumiaki Gochi for their contribution to the clinical procedure.

\section{References}

1. Shah RM, Spirn PW, Salazar AM, Steiner RM, Cohn HE, Solit RW, et al. Localization of peripheral pulmonary nodules for thoracoscopic excision: value of CT-guided wire placement. AJR Am J Roentgenol. 1993;161:279-83.

2. Dendo S, Kanazawa S, Ando A, Hyodo T, Kouno Y, Yasui K, et al. Preoperative localization of small pulmonary lesions with a short hook wire and suture system: experience with 168 procedures. Radiology. 2002;225:511-8.

3. Yoshida Y, Inoh S, Murakawa T, Ota S, Fukayama M, Nakajima J. Preoperative localization of small peripheral pulmonary nodules by percutaneous marking under computed tomography guidance. Interact Cardiovasc Thorac Surg. 2011;13:25-8.

4. Tsuchida M, Yamato Y, Aoki T, Watanabe T, Koizumi N, Emura I, et al. CT-guided agar marking for localization of nonpalpable peripheral pulmonary lesions. Chest. 1999;116:139-43.

5. Miyoshi K, Toyooka S, Gobara H, Oto T, Mimura H, Sano Y, et al. Clinical outcomes of short hook wire and suture marking system in thoracoscopic resection for pulmonary nodules. Eur J Cardiothorac Surg. 2009;36:378-82.
6. Lenglinger FX, Schwarz CD, Artmann W. Localization of pulmonary nodules before thoracoscopic surgery: value of percutaneous staining with methylene blue. AJR Am J Roentgenol. 1994;163:297-300.

7. Chino S, Kuriyama K, Isohashi K, Murata M, Mitani H, Tsuda K, et al. [Percutaneous localization of pulmonary nodules with $\mathrm{CT}$ guidance for lung resection: use of dyes]. Nihon Igaku Hoshasen Gakkai Zasshi. 2003;63:308-10.

8. Magistrelli P, D’Ambra L, Berti S, Feleppa C, Stefanini T, Falco E. Use of India ink during preoperative computed tomography localization of small peripheral undiagnosed pulmonary nodules for thoracoscopic resection. World J Surg. 2009;33:1421-4.

9. Sugi K, Kaneda Y, Hirasawa K, Kunitani N. Radioisotope marking under CT guidance and localization using a handheld gamma probe for small or indistinct pulmonary lesions. Chest. 2003;124:155-8.

10. Sortini D, Feo C, Maravegias K, Carcoforo P, Pozza E, Liboni A, et al. Intrathoracoscopic localization techniques: review of literature. Surg Endosc. 2006;20: 1341-7.

11. Horan TA, Pinheiro PM, Araujo LM, Santiago FF, Rodrigues MR. Massive gas embolism during pulmonary nodule hook wire localization. Ann Thorac Surg. 2002;73:1647-9.

12. Sakiyama S, Kondo K, Matsuoka H, Yoshida M, Miyoshi T, Yoshida S, et al. Fatal air embolism during computed tomography-guided pulmonary marking with a hook-type marker. J Thorac Cardiovasc Surg. 2003;126:1207-9.

13. Iguchi T, Yoshioka T, Muro M, Miyasho K, Inoue D, Hiraki T, et al. Systemic ai embolism during preoperative pulmonary marking with a short hook wire and suture system under CT fluoroscopy guidance. Jpn J Radiol. 2009;27:385-8.

14. Ohi S, Itoh Y, Neyatani H, Suzuki K, Kazui T. [Air embolism following computed tomography-guided lung needle marking: report of a case]. Kyobu Geka. 2004:57:421-3.

15. Kamiyoshihara M, Sakata K, Ishikawa S, Morishita Y. Cerebral arterial air embolism following CT-guided lung needle marking: report of a case. J Cardiovasc Surg (Torino). 2001;42:699-700.

16. Mizutani E, Nakahara K, Miyanaga S, Yoshiya T. [Hyperbaric oxygen therapy for air embolism complicating computed tomography (CT)-guided needle marking of the lung]. Kyobu Geka. 2012;65:899-902.

17. Iwasaki Y, Nagata K, Yuba T, Hosogi S, Kohno K, Ohsugi S, et al. Fluoroscopyguided barium marking for localizing small pulmonary lesions before videoassisted thoracic surgery. Respir Med. 2005;99:285-9.

18. Asano F, Shindoh J, Shigemitsu K, Miya K, Abe T, Horiba M, et al. Ultrathin bronchoscopic barium marking with virtual bronchoscopic navigation for fluoroscopy-assisted thoracoscopic surgery. Chest. 2004;126:1687-93.

19. Okumura T, Kondo H, Suzuki K, Asamura H, Kobayashi T, Kaneko M, et al Fluoroscopy-assisted thoracoscopic surgery after computed tomographyguided bronchoscopic barium marking. Ann Thorac Surg. 2001;71:439-42.

20. Endo M, Kotani Y, Satouchi M, Takada Y, Sakamoto T, Tsubota N, et al. CT fluoroscopy-guided bronchoscopic dye marking for resection of small peripheral pulmonary nodules. Chest. 2004; 125:1747-52.

21. Bando T, Yamagihara K, Ohtake Y, Miyahara R, Tanaka F, Hasegawa S, et al. A new method of segmental resection for primary lung cancer: intermediate results. Eur J Cardiothorac Surg. 2002;21:894-9; discussion 900.

22. Tsubota N, Ayabe K, Doi O, Mori T, Namikawa S, Taki T, et al. Ongoing prospective study of segmentectomy for small lung tumors. Study Group of Extended Segmentectomy for Small Lung Tumor. Ann Thorac Surg. 1998;66:1787-90.

23. Tomiyama N, Yasuhara Y, Nakajima Y, Adachi S, Arai Y, Kusumoto M, et al. CT-guided needle biopsy of lung lesions: a survey of severe complication based on 9783 biopsies in Japan. Eur J Radiol. 2006;59:60-4. 